\title{
Border Trap Characterisation by Measurements of Current-Voltage Characteristics of MOS Capacitors
}

\author{
Sima Dimitrijev, Philip Tanner, Z.-Qiang Yao, H. Barry Harrison
}

\begin{abstract}
Slow current transients in metal-oxidesemiconductor (MOS) capacitors have been observed and related to border traps (traps that are too slow to be classified as interface traps, and to fast to be classified as oxide traps). This paper describes border-trap related current transients induced by voltage steoping as a possible technique for both energy-level and time-response characterisation of the border traps. The voltage stepping measurements are compared to the standard linear voltage ramping technique.
\end{abstract}

\section{INTRODUCTION}

A number of charge traps appear at and around the interface between silicon and silicon dioxide. The techniques used to characterise these traps generally provide energy distribution of trap density, ignoring timeresponse characteristics of the traps. These techniques are quite acceptable for the traps that are charged and discharged very quickly (generally known as interface traps), or the traps that are too slow to possibly change their charge during standard $I-V$ and $C-V$ measurements (generally known as oxide traps). However, there is a class of traps known as border traps[1], [2], with time responses that need to be precisely characterised, as they are neither too short (like the interface traps) nor too long (like the oxide traps).

We recently reported on slow current transients in metal-oxide-semiconductor (MOS) capacitors[3], and suggested that these transients could be used to characterise the border traps[4]. We obtained the slow current transients using an HP4145B semiconductor parameter analyzer which scans the voltage in a staircase fashion (referred to as stepping in this paper) and measures the current at a set delay time following each voltage step, thus ignoring any fast displacement currents. In this paper, we provide a further insight into the current transients in MOS capacitors obtained by voltage stepping, and discuss the voltage stepping as a simple technique to provide both the energy and time distribution of the border trap density.

S. Dimitrijev, P. Tanner, and H.B. Harrison are with the School of Microelectronic Engineering, Griffith University, Nathan, Brisbane, Queensland 4111, Australia; e-mail: sima@me.gu.edu.au

Z.-Q. Yao is with AWA Microelectronics, 8 Australia Ave., Homebush, NSW 2140, Australia

\section{EXPERIMENT}

Different dielectrics, gate materials, and substrate dopings were used to measure the current transients. The structures used include: (i) MOS capacitors with polycrystalline silicon gates, and $8 \mathrm{~nm}$ and $40 \mathrm{~nm}$ silicondioxide dielectrics on p-type silicon, fabricated in AWA Microelectronics, Homebush, NSW, by a standard CMOS technology process, and (ii) Al-gate MOS capacitors on $\mathrm{n}$ - and p-type silicon with oxynitride dielectrics (nitrided in either $\mathrm{NO}$ or $\mathrm{N}_{2} \mathrm{O}$ ambient), fabricated in our laboratory. The results shown in the graphs in this paper are for the oxides grown on $<100>1.4-5.0 \Omega \mathrm{cm}$ n-type silicon wafers in pure oxygen at $1000^{\circ} \mathrm{C}$, and then annealed in nitric oxide at $1150^{\circ} \mathrm{C}$ for $5 \mathrm{~min}$ [5]. The dielectric thickness in these samples was $4.5 \mathrm{~nm}$, while the area of the aluminium gates was $0.01 \mathrm{~cm}^{2}$.

Current-voltage characteristics of the MOS capacitors are measured by both the above-mentioned stepping technique and the standard linear ramping technique to enable comparative study. To obtain the current-voltage characteristics, HP4145B semiconductor parameter analyzer ramps the voltage in a staircase fashion. This procedure was directly used as the stepping technique. The voltage step size used in this experiment was $10 \mathrm{mV}$. The time step size consists of two parts: delay time which is set by the user, and a measurement time which is $60 \mathrm{~ms}$ when currents smaller than $1 n A$ are measured [6]. Different delay times were used in this experiment in order to vary the stepping rate. The results shown in the graphs are for $0 s$ delay time.

The HP4145B semiconductor parameter analyzer was also used to measure the current by the linear ramping technique. In this case, however, the voltage was linearly ramped at a rate of $100 \mathrm{mV} / \mathrm{s}$ by an independent function generator. This technique is widely used and known as quasi-static capacitance-voltage (C-V) measurement technique. To obtain the quasi-static capacitance, the measured current $I$ is divided by the ramp rate: $C=I / \frac{d V}{d t}$. In this paper, the current $I$ in addition to the capacitance $C$ is shown in the graph to compare with the current obtained by the stepping technique.

Finally, high-frequency $(100 k H z) \mathrm{C}-\mathrm{V}$ characteristics were also measured by an HP4284A LCR meter. 


\section{RESULTS AND DISCUSSION}

Typical current-voltage characteristics obtained by the stepping technique (solid line) and the linear ramping technique (dashed line) are shown in Fig. 1 along with the corresponding high-frequency $C-V$ curve (dotted line).

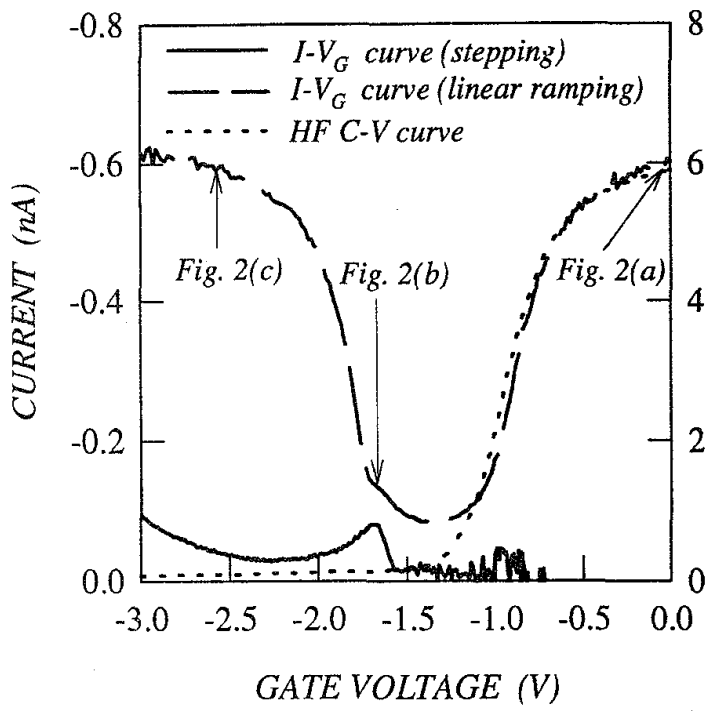

Fig. 1. Comparison of the current-voltage :haracteristics of MOS capacitor obtained by voltage stepping (solid line) and linear voltage ramping (dashed line). The dashed line also expresses the quasi-static capacitance, which can be compared to the high-frequency capacitance shown by the dotted line.

The voltage is ramped/stepped from $0 \mathrm{~V}$ to $-3 \mathrm{~V}$. At around $O V$ the n-type substrate of the MOS capacitor is in accumulation, and the linear voltage ramping produces a constant negative current (about $-0.6 n \mathrm{~A}$ ) due to electron flow from the surface into the bulk of the silicon substrate. The energy-band diagram and the voltage/current time dependencies shown in Fig. 2 (a) illustrate this situation. Voltage stepping, also illustrated in Fig. 2 (a), produces spikes of current. As the time constant of these spikes is very short, the transient current dies away before the current measurement is taken after time $t_{\text {step. }}$. As a consequence, zero current is recorded by the stepping technique as opposed to the constant current of $-0.6 n A$ recorded by the linear ramping technique.

As the negative voltage is increased, the surface of the substrate is first depleted of the majority electrons and subsequently minority holes are attracted (weak inversion). The depletion process leads to a capacitance drop which can be observed on both, the high-frequency $C-V$ curve and the linear ramping current (equivalent to the quasi-static $C-V$ curve). No current is recorded by the stepping technique, as the current transients are shorter than the measurement time step $t_{\text {step }}$. It is obvi- ous that the stepping technique filters out the fast current transients due to the capacitor displacement current. The weak inversion process increases the capacitance again, which results in the increase of the linearramping current observed in Fig. 1 beyond $-1.4 \mathrm{~V}$, while the high-frequency capacitance remains low as it is insensitive to the increase in the density of the minority carriers. Careful inspection of the linear-ramping current (the dashed line in Fig. 1) discovers a distortion at around $-1.7 \mathrm{~V}$. Typically, such a distortion is due to the effect of interface or near-interface traps. The energy-band diagram and the voltage/current time dependencies shown in Fig. 2 (b) illustrate the effects at around $V_{G}=-1.7 \mathrm{~V}$. While it is theoretically possible to extract the current due to the traps (the distorting current) from the total linear-ramping current, this curve provides no information about the time response of the traps involved. The current measured by the stepping technique (the solid line in Fig. 1) shows a peak at around $V_{G}=-1.7 \mathrm{~V}$. The current response to the voltage stepping, shown in Fig. 2 (b), illustrates that the current measured by the stepping technique is due to very slow transients, labelled as process 2 . The band diagram of Fig. 2 (b) illustrates that the slow transients are due to the electrons being emitted from the border traps appearing above the Fermi level (process 2 ) as the gate voltage is stepped down.

As the voltage is stepped towards more negative values, the strong inversion region is reached, and the ramping current flattens out again at about $-0.6 n \mathrm{~A}$, which is the charging current needed to maintain $100 \mathrm{mV} / \mathrm{s}$ voltage change across the gate oxide capacitance. This situation is illustrated in Fig. 2 (c). As all the border traps are assumed to be above the Fermi level at this gate bias, the voltage stepping does not produce the long current transients any longer, and the current recorded by the stepping technique is expected to drop to zero. This situation is indeed observed in many samples that we analysed. The graph shown in Fig. 1 is for a very thin gate oxide $(4.5 \mathrm{~nm})$, and the stepping current (the solid line) observed at high negative voltages is a leakage current due to electron tunneling through the oxide.

Fig. 1 and Fig.2 show that the stepping technique can be used to automatically extract the border-trap induced current transients from the ordinary transients due to the charging/discharging of the MOS capacitor. This is achieved by adjusting the time step $t_{s t e p}$ so that the current transient due to the generation of the minority carriers, which is typically assisted by the interface traps, dies away before the current measurement is taken. In other words, the stepping technique can be made insensitive to the transients due to the minority carrier generation, as it is insensitive to any transients that are completed within $t_{\text {step }}$. Actually, the stepping technique measures only the transient current 

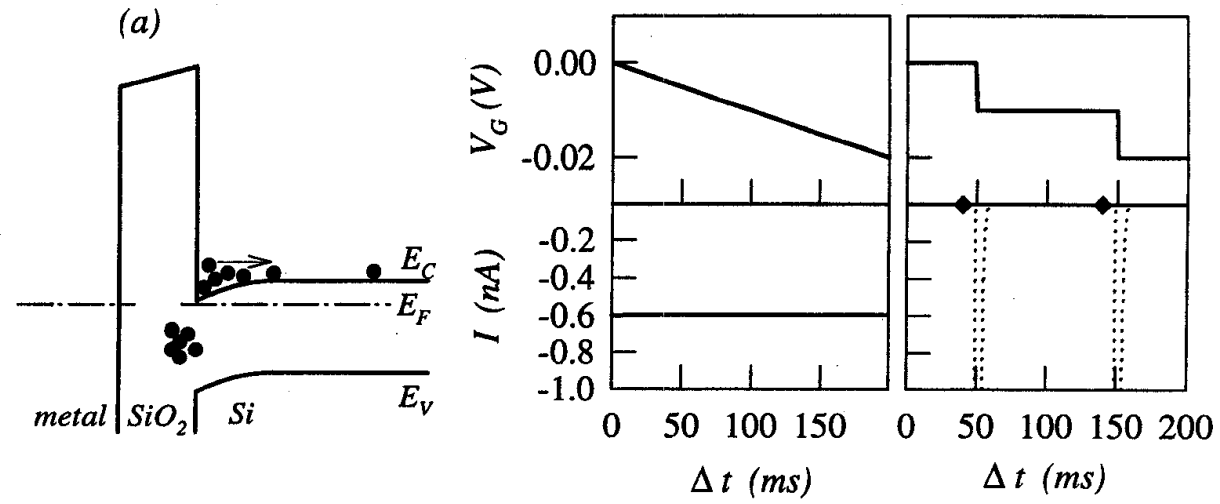

(b)
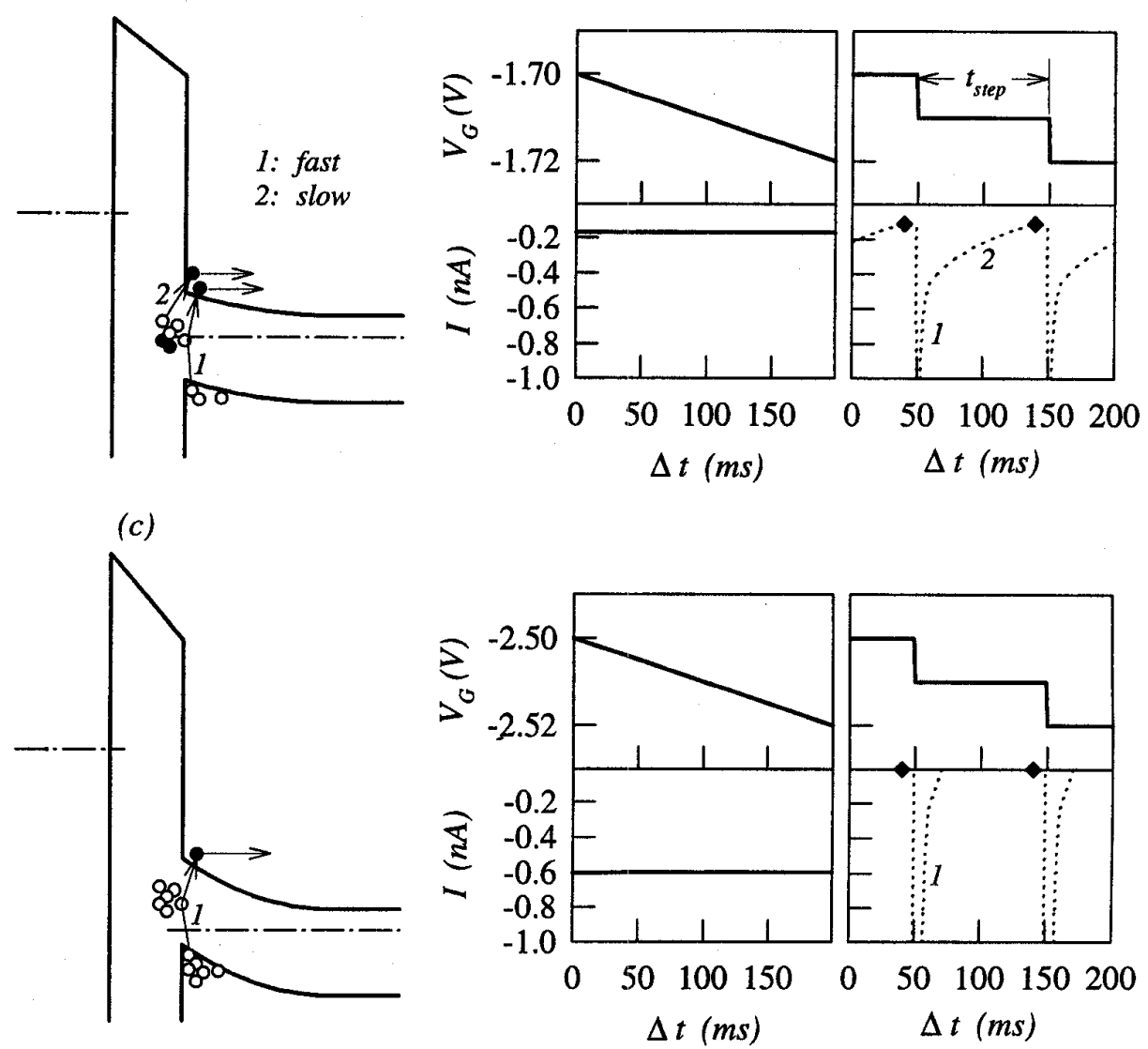

Fig. 2. Comparison between the linear ramping and the stepping technique. The second and third columns illustrate the current transients produced by linear voltage ramping and voltage stepping, respectively. The band diagrams in the first column illustrate the involved current-transient mechanisms: 1 - minority carrier generation, 2-border trap discharging. The graphs under (a), (b) and (c) represent the cases of $V_{G}=0 \mathrm{~V}, V_{G}=-1.7 \mathrm{~V}$, and $V_{G}=-2.7 \mathrm{~V}$, respectively. 
at time $t=t_{\text {step }}$, that is the border traps that are discharged at time $t=t_{\text {step }}$. This means that repeated voltage stepping with scanned $t_{\text {step }}$ values would provide direct measurements of the time distribution of the border traps. The measured current can be converted into border trap density in a straightforward way. Also, the gate voltage $V_{G}$ can be converted into the Fermi level position by some of the existing and well established techniques. Therefore, the stepping technique described can be used to provide two-dimensional distribution (that is energy-band and time distribution) of the border traps.

It should be noted that the generation of the minority carriers (labelled as process 1 in Fig. 2 b) limits the minimum response time of the border traps that can automatically be extracted. With this in mind, the minimum value of the time step $t_{\text {step }}$ should be carefully chosen to avoid interference of the transient current from the previous step with the measurement of the transient current from the last (active) step. Finally, note that it is also possible to step the voltage from negative to positive values, in which case charging of the border traps is monitored.

\section{CONCLUSIONS}

Border traps are known to produce slow current transients with time constants ranging from milliseconds to hundreds of seconds. The standard linear ramping technique used to induce a transient current and to enable quasi-static capacitance measurement does not provide any information on the time behaviour of the border traps.
This paper shows that voltage stepping with different time step sizes can be used to effectively measure the density of the border traps with different time constants. This enables the border trap density to be expressed as a two-dimensional function of time response and energy position. This makes the characterisation of the border traps more detailed than the characterisation by way of energy distribution of the trap density which is used in the case of the interface traps. The inclusion of the time dimension in the case of the border traps is necessary, and can further be developed into position (distance from the interface) distribution.

\section{REFERENCES}

[1] D.M. Fleetwood, M.R. Shaneyfelt, and J.R. Schwank, "Estimating Oxide Trap, Interface Trap, and Border Trap Charge Densities in Metal-Oxide- Semiconductor Transistors," A ppl. Phys. Lett., vol. 64, no. 15, p. 1965, 1994.

[2] D.M. Fleetwood, P.S. Winokur, R.A. Reber, Jr, T.L. Meisenheimer, J.R. Schwank, M.R. Shaneyfelt, L.C. Riewe, "Effects of Oxide Traps, Interface Traps, and 'Border Traps' on Metal-Oxide-Semiconductor Devices," J. Appl. Phys. vol. 73 , no. 10, pp. 5058-5074, 1993.

[3] Z.-Q. Yao, S. Dimitrijev, P. Tanner, and H.B. Harrison, "Slow Current Transients in Metal-Oxide-Semiconductor Capacitors," Appl. Phys. Lett., vol. 66, no. 19, pp. 25102512, May 1995.

[4] P.G. Tanner, S. Dimitrijev, and H.B. Harrison, "Evidence of Border Traps in Wet Etched and Plasma Eitched MOS Capacitors," in Proc. 13th Australian Microelectronic Conference, Adelaide, Australia, July, 1995, pp. 56-61.

[5] Z.-Q. Yao, H.B. Harrsion, S. Dimitrijev, and Y.-T. Yeow, "Effects of Nitric Oxide Annealing of Thermally Grown Silicon Dixide Characteristics," IEEE Electron Dev. Lett., vol. 16, no. 8, pp. 345-347, August 1995.

[6] HP4145 Semiconductor Parameter Analyzer, Operating manual, Hewlett-Packard, 1989. 